\title{
Distributed QoS Constraint based Resource Adaptation Strategy for Cognitive Radio Networks
}

\author{
Yakubu S. Baguda \\ Information Systems Department, \\ Faculty of Computing and Information Technology, \\ King Abdulaziz University, Saudi Arabia.
}

\begin{abstract}
This paper primarily investigates and addresses the optimal power adaptation strategy problem for multi-user cognitive radio network. The need to determine the optimal power transmission for the secondary user (SU) in a distributed network environment is challenging and important. This requires an efficient adaptive strategy with high convergence capability in order to meet up with the multi-users quality of service $(\mathrm{QoS})$ requirements in a cognitive network and ultimately ensure more efficient resource utilization amongst users. In this paper, a distributed QoS contraint scheme which considered both the transmission power and outage probability has been proposed to maximize the network performance and to control the SUs transmission power. Firstly, the QoS constraint optimization problem for distributed secondary users is formulated and solved in order to adapt with the network dynamics of the cognitive networks. Subsequently, the solution has been used to dynamically adjust the radio power of the SUs to conform with the stringent network constraint and ensures coexistence amongst the users. More importantly, the simulation result shows that the scheme increases the overall network utility when compared to the scheme without adaptation.
\end{abstract}

Keywords-Cognitive radio network; outage probability; quality of service $(Q o S)$; network utility; power adaptation; primary user; secondary user

\section{INTRODUCTION}

There has been explosive growth in mobile devices market and high bandwidth demand which eventually leads to more congestion and traffic within the frequency spectrum. It is known fact that the spectrum is primarily allocated to primary users (PU), but has not been utilized effectively. Hence, making usage of the available resources in effective manner requires an efficient resource allocation and transmission strategy to dynamically adapt with the network condition. Therefore, there is dramatic need for highly adaptive schemes to efficiently manage the spectrum utilization. This eventually led to development of cognitive radio technology in order to efficiently and effectively manages the spectrum [1]. Sensing for free spectrum is challenging and it is very important in cognitive radio as such the decision depends on the precision to determine the spectrum availability or presence of the primary user. The performance of the secondary user can be significantly improved by determining the available spectrum that can be used regularly by the secondary user if condition warrant. When more users are involved in the decision, the probability of false alarm is higher, but at the expense of increasing the latency.
As it has previously been mentioned, making effective decision is essential which can lead to higher performance and reliable communication in cognitive radio network. More importantly, efficient power level sensing mechanism will improves the performance and QoS provision by exactly determining the presence of PU and the network utilization. For instance, the network condition rapidly changes and hence the decision time should be negligible and precise as well to ensure effective utilization of the network. The ability to determine the probability of false alarm precisely matters lot while taking decision. It is challenging to reduce the decision delay time especially in a network with large number of users and at the same time achieving optimal performance. Hence, there is need for high effective optimization scheme in order to achieve high performance and conforming to the power constraint in which every SU is expected to abide by. Managing and utilizing the available limited resources will increase the network performance, capacity and reduces the complexity. In most of the literature works, the users were assumed to have the same features and setting or uniform parameters. In real world scenario, the devices have different sensing parameter and requirements. This should be considered while designing such systems. Designing schemes to combine the collective sensing information from different devices and ensuring effective decision with sophistication and precision is extremely important for effective communication to harness.

Different research works related to dynamic spectrum sensing have been conducted in order to improve the throughput of the SU and at the same time protecting the PU [2], [3]. It is known fact that the probability of false alarm depends greatly on the sensing parameters and throughput as well. In [4], the resource allocation by maximizing the total throughput of the secondary user has been exploited. More importantly, both sensing time and power were considered for enhancing the cognitive radio performance. Some researches related to spectrum sharing have been focusing on how to used power constraint on secondary users not exceeding the maximum transmission power level so that not to interrupt with the primary user operation [5]-[7]. Consequently, this will reduce the intereference in the cognitive radio network and enhence performance.

Section 2 will cover the related works. Section 3 mainly focuses on the system model and problem formulation. Section 4 discusses the developed distributed QoS constraint based power adaptation scheme. Numerical results and 
discussion for experimental simulation is presented in Section 5. Finally, the conclusions for the research are drawn in Section 6.

\section{RELATED WORK}

Distributed power control has been studied in [8] and the scheme mainly ensures that the feasible solution meet up with the requirement of the users. A joint optimization scheme for cell selection and power control was proposed [9]. Huang et al. [10] used power price and interference to adjust power transmitted by the users with the multi-hop wireless network. In [11], both flow rate and transmitted power were used in order to achieve high network utilization. It assumed static channel condition in which is not possible in real world scenario. A centralized power control scheme using outage probability in [12] has been proposed and it considers the channel fading. In [13], it has formulated the QoS optimization problem in a multi-user environment.

An auction based power allocation scheme was developed in [14] to share spectrum amongst distributed users but it has taken the interference temperature into consideration. Srinivasa et al. [15] studied joint beam forming and power control scheme for different users with multiple antennas.

Wang et al. [16] considered the interference amongst the secondary users and used an exponential pricing function to protect the primary users from interference. More importantly, it considered many secondary and primary users which use the same band for their operation. In [17], multiple users and single PU were considered in the distributed allocation problem scenario in order to minimize the transmitted power. Mei et al. [18] proposed a fast convergence distributed power control algorithm which uses round robin. In [19], it uses game theory to minimize the sum throughput of the SUs.

Hence, it is very important to note that the developed scheme is different from other power control scheme because it primarily considers the maximum transmission power and the QoS needed by PUs and SUs for reliable and effective communication. More importantly, the developed scheme maximize the overall cognitive radio network spectral utility and at the same time conforming to the general coexistence policy of the network in such a way that the primary users are not interrupted or interfered by the secondary users.

\section{SYSTEM MODEL AND PROBLEM FORMULATION}

In this section, the system model used for the distributed QoS constraint based power adaptation scheme has been described. The scenario setting shown in Fig. 1 has been used for the multi-user cognitive radio network. Firstly, let's assume that there are $\mathrm{N}$ primary and $\mathrm{M}$ secondary users within the network. Also, the basic assumption for the development of the model are introduced in order to have clear understanding of the general assumptions and setting used for the development of the proposed scheme.

In order to model the QoS constraint power adaptive scheme for the above cognitive radio network, it is assumed that there secondary transmitter (SUBs) which is within the coverage area of the cognitive network. Also, it is assumed that the transmitted power by $\mathrm{N}$ secondary users can be represented mathematically as follows:

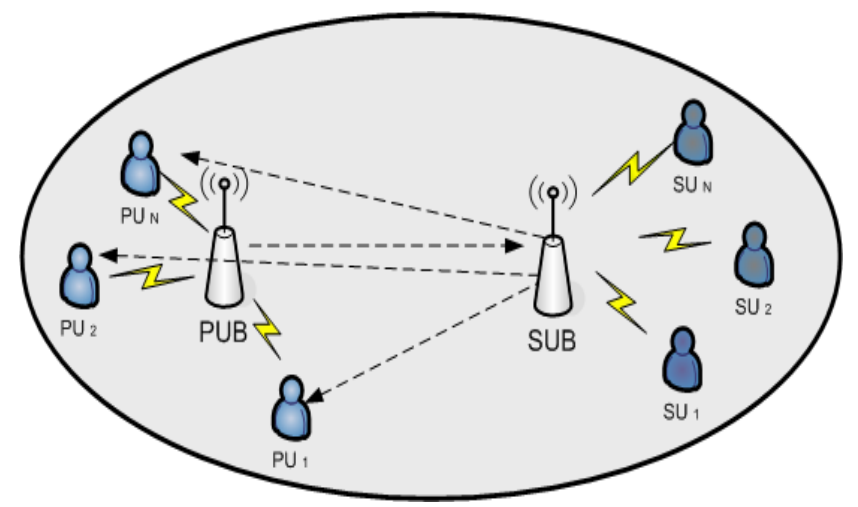

Fig. 1. A typical multi-user cognitive radio network scenario.

$$
\mathrm{P}_{\mathrm{i}}=\left[\mathrm{P}_{1}, \cdot \cdot \cdot \cdot \mathrm{P}_{\mathrm{N}}\right]
$$

The channel utilization is assumed to be slotted and the secondary users synchronize their spectrum access based on the time slot. In order to avert collision, every node ensures that it listen to the channel before transmit. In this work, the spectral utility of secondary users is determined using the mathematical equation shown as follows:

$$
\mathrm{U}_{\mathrm{i}}=\log \left(1+\mathrm{h}_{\mathrm{i}} \mathrm{P}_{\mathrm{i}}\right)
$$

Where $h_{i}$ represent the constant taking care of the parameters such as coverage, intereference for the secondary users (i.......... ). The utility is connected or related to the information rate which can be effectively conveyed over the secondary user link at a particular time. Ultimately, it varies with the change in network condition as in the real world scenario. To ensure coexistnce amongst the users within the network, the power transmitted by users $\mathrm{P}_{\mathrm{i}}$ should be less than the maximum power $\mathrm{P}_{\max }$. So all the users have to abide with the maximum power threshold in order to prevent other users interefering the primary users.

Lets assumed that No is the gussian noise at PUB and hence the SINR due to channel can be computed using the equation below:

$$
\beta=\frac{P_{0} G_{0} F_{0}}{N_{0}+\sum_{i=1}^{N} P_{i} G_{i} F_{i}}
$$

Assuming $\mathrm{F}_{0}$ is exponentially distributed and the outage probability of the PUB is given by

$$
\emptyset_{0}=1-\exp \left[-\frac{N_{0} \beta_{t h}}{P_{0} G_{0}}\right]
$$

$\beta_{t h}$ present the required SINR and it ultimately ensures that both the QoS requirements of the PU and SU have been achieved.

In order to develop the optimization problem needed to meet up the power and outage probability. The primary objective is to maximize the secondary user utility function given in (5) and subsequently taking into consideration the power and outage probability constraint as well. The 
optimization problem can be mathematically described as follows:

$$
\begin{aligned}
& \text { Maximize } U=\sum_{i=1}^{M} \log \left(1+h_{i} P_{i}\right) \\
& \text { Subject to: } \\
& \begin{aligned}
\sum p_{i} \leq p_{\max } & \forall i \\
\sum \emptyset_{i} \leq \emptyset_{\max } & \forall i
\end{aligned}
\end{aligned}
$$

In the optimization problem presented in (5), the outage probability and transmission power have been considered as constraints need to be fulfilled by the optimization problem. The SU utility which satisfied the constraint is selected as the utility values to conform to the PU and SU QoS requirement. The value of $\mathrm{p}$ and $\emptyset$ for SUs $(i=1 \ldots \ldots \ldots . M)$ is compared with the maximum value in order to ensure that the PU and SU coexist within the same coverage without interference amongst the users. In order to achieve optimal utility while considering the QoS constraint, (5) has been used in optimizing the SU performance. In the next section, a detail about the QoS constraint power adaptive algorithm has been described.

\section{QOS CONSTRAINT POWER ADAPTIVE AlgORITHM}

The main primary objective is to reduce the outage probability by maximizing the utility of the secondary users within the cognitive radio network. Initially, the problem has been formulated as convex optimization and its closed solution is derived. The algorithm is used to approximate the optimal solution based on the transmission power and outage probability of the secondary users in the cognitive radio network. The dynamics of the secondary users with the cognitive radio network can be handle using heuristics - that is to learn and improve with time. Regularly, the SU's estimate the outage probability and transmission power in order to tackle the rapid change in the network condition.

The distributed QoS constraint based power adaptation algorithm is as shown below and the parameters used by the algorithm are initialized. Having initialized the constant parameters, others parameters such as $\mathrm{p}, \mathrm{q}$ are computed by users as the time increase from 0 to $\mathrm{t}(0 \rightarrow t)$. The main key issue of concern is the fact that how the distributed QoS constraint based power algorithm will meet up with both power and outage probability constraints. The SUs receives information from the SUB about the network dynamics and based on that it decides on either to utilize the PU spectrum.

Algorithm 1 Distributed QoS Constraint Based Power Adaptation Scheme

01. Set value for $\mathrm{N}_{0}, p_{\max }, \emptyset_{\max }$, number of SUs

02. Calculate $p_{i} \& \emptyset_{i}$ for each SU

03. while $(\mathbf{i}<\mathbf{M})$ do

04. If $\left(\left(\emptyset_{i} \geq \emptyset_{\max }\right) A N D\left(0 \leq p_{i} \leq p_{\max }\right)\right)$

05. $\mathbf{U}_{\mathbf{i}}=\max \left(\sum_{i=1}^{M} \log \left(1+h_{i} P_{i}\right)\right)$

else if $\left(p_{i} \geq p_{\max }\right)$

06.

$p_{i}=p_{\max }$

07. Update $\mathrm{U}_{\mathrm{i}}{ }^{\mathrm{n}+1}$ and $\mathrm{P}_{\mathrm{i}}{ }^{\mathrm{n}+1}$
08. Go to step 5
09. end if
10. end if
11. $i=i+1$
12. end while

Initially, the values for $p_{\max }$ and $\emptyset_{\max }$ are set as the constraint need to be satisfied before determining the network utility $\mathrm{U}_{\mathrm{i}}$ for any $\mathrm{SU}$. They represent the power and outage probability threshold in which all the users have to conform with. In a network with $M$ secondary users, each user while updating its power has to compare with the maximum power threshold. The transmitted power for SUs should always be less than $p_{\max }$ in order not to interfere with other users. The values $p_{i} \& \emptyset_{i}$ for each SU are computed and tested to ensure that they meet up with the constraint. If the condition has been satisfied, the network utility is computed based on (5). If $p_{i} \geq p_{\max }$, the maximum power threshold value is chosen as the value the SU should use as its transmission power level. The SU decides by juxtaposing the $\mathrm{P}_{\mathrm{i}}$ with the $\mathrm{P}_{\max }$. When $\mathrm{P}_{\mathrm{i}}$ $<\mathrm{P}_{\max }$, the $\mathrm{SU}$ will automatically access the spectrum and it transmit with the power $\mathrm{P}_{\mathrm{i}}$. Also, it considers the outage probability threshold as well. Otherwise, the SU does not engage in to any activity with the PU spectrum. In Step 7, both network utility and power for the secondary user are updated. By repeating this procedure over time, the algorithm converges to the peak power level and this dramatically improves the network utility since $\mathrm{P}_{\mathrm{i}}$ is directly related to it.

Since the develop algorithm has restriction on SUs knowing about other SUs activities, they only intermingle with the SUB unit. In a very large network which has many SUB and PUB, each SUB has information about all the SUs and PUBs connected to it. This requires more strategic approach to effectively sense the condition of the network and eventually fulfill the constraints thresholds under such situation. Each SU decide independently whether to utilize the spectrum opportunity or not. Once it has been decided, the SU will access or share information via the available medium.

Based on (5), the problem can be further expressed and represented in Lagrange form by

$$
\begin{array}{r}
\boldsymbol{L}(p, \emptyset, \lambda)=\sum_{i} \log \left(1+h_{i} p_{i}\right) \\
-\sum_{i} \lambda_{i}\left[\left(\sum_{i} p_{\max }-p_{i}\right)\right. \\
\left.+\left(\sum_{i} \emptyset_{i}-\emptyset_{\max }\right)\right] \\
=\sum_{i} \log \left(1+h_{i} p_{i}\right)+\sum_{i} \lambda_{i}\left(\sum_{i} p_{i}-p_{\max }\right) \\
+\sum_{i} \lambda_{i}\left(\sum_{i} \emptyset_{i}-\emptyset_{\max }\right)
\end{array}
$$




$$
\begin{aligned}
& =\sum_{i} \log \left(1+h_{i} p_{i}\right)+\sum_{i} \lambda_{i} \sum_{i} p_{i}-\sum_{i} \lambda_{i} p_{\max } \\
& +\sum_{i} \lambda_{i} \sum_{i} \emptyset_{i}-\sum_{i} \lambda_{i} \emptyset_{\max } \\
& =\sum_{i} \log \left(1+h_{i} p_{i}\right)+\sum_{i} \lambda_{i} \sum_{i} p_{i}+\sum_{i} \lambda_{i} \sum_{i} \emptyset_{i} \\
& -\sum_{i} \lambda_{i} p_{\max }-\sum_{i} \lambda_{i} \emptyset_{\max } \\
& =\sum_{i}\left(\log \left(1+h_{i} p_{i}\right)+\lambda_{i} \sum_{i} p_{i}+\lambda_{i} \sum_{i} \emptyset_{i}\right) \\
& -\sum_{i} \lambda_{i} p_{\max }-\sum_{i} \lambda_{i} \emptyset_{\max }
\end{aligned}
$$

If $q_{i}=\lambda_{i} \sum_{i} p_{i}+\lambda_{i} \sum_{i} \emptyset_{i}$, the equation above can be rewritten as

$$
\begin{gathered}
=\sum_{i}\left(\log \left(1+h_{i} p_{i}\right)+q_{i}\right)-\sum_{i} \lambda_{i} p_{\max } \\
-\sum_{i} \lambda_{i} \emptyset_{\max }
\end{gathered}
$$

Where $\mathrm{q}=\left[\mathrm{q}_{1}, \mathrm{q}_{2}, \ldots \ldots . \mathrm{q}_{l}\right]$ and hence the Lagrange of the dual function to the optimization problem can be represented mathematically as follows:

$$
\begin{gathered}
G(p, \emptyset, \lambda)=\min (L(p, \emptyset, \lambda)) \\
G(I, x, \lambda)=\min (L(I, x, \lambda)) \\
=\min \left(\sum_{i}\left(\log \left(1+h_{i} p_{i}\right)+q_{i}\right)-\sum_{i} \lambda_{i} p_{\text {max }}-\right. \\
\left.\sum_{i} \lambda_{i} \emptyset_{\text {max }}\right)
\end{gathered}
$$

By setting (9) to zero and re-arranging the terms in the equation, it eventually yields

$$
\begin{aligned}
\sum_{i}\left(\log \left(1+h_{i} p_{i}\right)\right. & \left.+q_{i}\right) \\
& =\sum_{i} \lambda_{i} p_{\max }+\sum_{i} \lambda_{i} \emptyset_{\max }
\end{aligned}
$$

The update of the dual variables is achieved using (11) and (12) as shown below:

$$
\begin{array}{cc}
p_{i}^{(n+1)}=\min \left\{p_{i}^{n}-\theta^{n}\left(p_{\max }-\sum_{i} p_{i}\right)\right\}, & \forall i \in I \\
\emptyset_{i}^{(n+1)}=\min \left\{\emptyset_{i}^{n}-\theta^{n}\left(\emptyset_{\max }-\sum_{i} \emptyset_{i}\right)\right\}, & \forall i \in I
\end{array}
$$

\section{A. Optimal Utility}

The set of optimal utility for each SU at a particular time t is determined and the best utility is selected based on the constraints. The set of utility for the secondary users can be represented mathematically as follows:
$\mathrm{U}_{\mathrm{i}}=\left[\mathrm{U}_{1}, \cdot \cdot \cdot \cdot, \mathrm{U}_{\mathrm{N}}\right]$

More importantly, it has been assumed that there are $\mathrm{N}$ secondary users within the cognitive radio network. For each secondary user, it optimal transmission and its required QoS is considered while selecting the best utility. Hence, equation can be further represented in simple form as follows:

$$
\mathrm{U}_{\mathrm{i}}=\left[\mathrm{U}_{1}\left(\mathrm{P}_{1}, \mathrm{Q}_{1}\right) \cdot \cdot \cdot \mathrm{U}_{\mathrm{N}}\left(\mathrm{P}_{\mathrm{N}}, \mathrm{Q}_{\mathrm{N}}\right)\right]
$$

Similarly, the outage probability for the secondary users is represented by equation (15) as follows:

$$
\emptyset_{\mathrm{i}}=\left[\emptyset_{1}, \cdot \cdot \cdot, \emptyset_{\mathrm{N}}\right]
$$

For every SU, the outage probability varies over period of time t. It can be noticed that $\emptyset_{\mathrm{i}}$ vary randomly below and above the threshold red line as shown in Fig. 2. The outage probability should not be fixed, but changes as the network condition changes. This is primarily to ensure that the simulation scenario mimic the real network scenario as well. In addition, the outage probability is affected by the distance, signal strength, noise and any other factor which can change the network condition. Each SU compare its outage probability with the threshold value in order to decide on whether to increase or decrease its transmission power and also by taking into consideration the maximum power threshold.

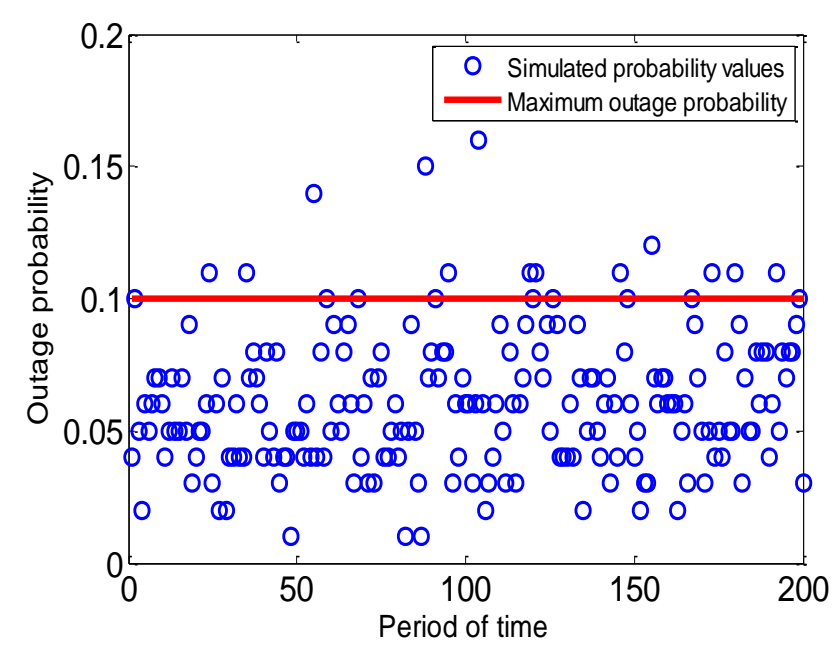

Fig. 2. Constraint outage and simulated outage probabilities.

As can be seen from Fig. 2, it shows the SUs outage probabilities variation over a period of time t. The developed scheme adapts with the change in outage probability and SU transmission power. In a nutshell, the primary motive for setting the outage probability threshold is to ensure that the SU QoS requirements have been accomplished for effective utilization of the network. For every SU, its outage probability is different from other $\mathrm{SU}$ as in real world scenario - this is mainly due to the aforementioned reason. The SU will decide to increase its transmission power whenever its outage probability is below the threshold value. More importantly, the SUs update their outage probability based on (12). This strategy helps a lot in making effective utilization of the network amongst the secondary users within the cognitive radio network. 


\section{SimUlation RESUlts AND ANALYSis}

This section primarily focus on the simulation results obtained from the proposed distributed QoS constraint based power control algorithm are presented and discussed in more details about the findings. As it has been mentioned that a multiple SUs were deployed within the cognitive radio network as shown in Fig. 1. The parameter settings used for the simulation are represented in Table I as shown below.

TABLE I. Simulation Parameter Settings

\begin{tabular}{|l|l|}
\hline Parameter & Value \\
\hline Path loss exponent & 3.5 \\
\hline PU transmission power & $2000 \mathrm{mw}$ \\
\hline SU maximum transmission power & $1000 \mathrm{mw}$ \\
\hline Number of SUs & 5 \\
\hline Outage probability & $10 \%$ \\
\hline
\end{tabular}

More importantly, the outage probability threshold for the PU has been set to $10 \%$ as constraint and it has ultimately been maintained constant throughout the period of time. The main primary key objective is to maximize the SU utility and enhance the overall network performance. Initially, the proposed distributed QoS constraint based power control scheme has been tested to verify that the outage probability does not remain constant but it varies over period of time as in real time scenario. Also, it is very clear that the SU's outage probability can above or below the threshold value and hence if it has not been properly managed, the QoS of the SU and PU can be significantly affected since there is every tendency that the users would interfere with one another.

\section{A. SUs Power Convergence}

Interestingly, the transmitted power by each SU has been a major issue of concern in the distributed QoS constraint based power control algorithm. Firstly, the power transmitted by each is critically analyze in order to ensure that all the SUs operated within the allowable power limit. As it can be seen in Fig. 3, the transmitted power by each user is far below the allowable threshold power level. More importantly, it can be noticed that all the users have different transmission power which is similar to the real world scenario. The power threshold for the SUs is $50 \%$ less than that of the PU. This is mainly to ensure that the SUs do not interfere with the PU while operating. For instance, the SU with the highest power is the secondary user 3 and then followed by 2, 1, 4, and 5 as shown in Fig. 3.

Even though that the distributed QoS constraint based power control scheme is decentralized system, coordinating and managing such scheme is challenging and it require thorough testing and evaluation to verify whether the performance of the developed scheme conform the requirements needed to be fulfilled. This requires systematic approach in order to efficiently test the scheme performance and its decision making precision. In order to critically analyze the performance of the developed power control scheme, the performance of each secondary user is evaluated since it is decentralized power control scheme. All the five (5) secondary users were considered so that each user conforms to the maximum power threshold. Based on the numerical experimentation, it can be seen from Fig. 2 that as time $t$ increases; the SUs adjusted their respective transmission power in order to achieve the optimal transmission power.

As it has been explained, each SU has different proximity from both the PUB and SUB. This has had dramatic impact on the SUs transmission power and coverage. As the time varies from 0 to any value of $t$, the transmitted power for SUs is determined and subsequently each SU updated its power level. As time increases, the power transmitted by each SU becomes relatively constant when the algorithm completely converges. This approach has been used to keep in line with the variation in network condition. Consequently, this improves the network utilization and it ensures fairness amongst the SUs.

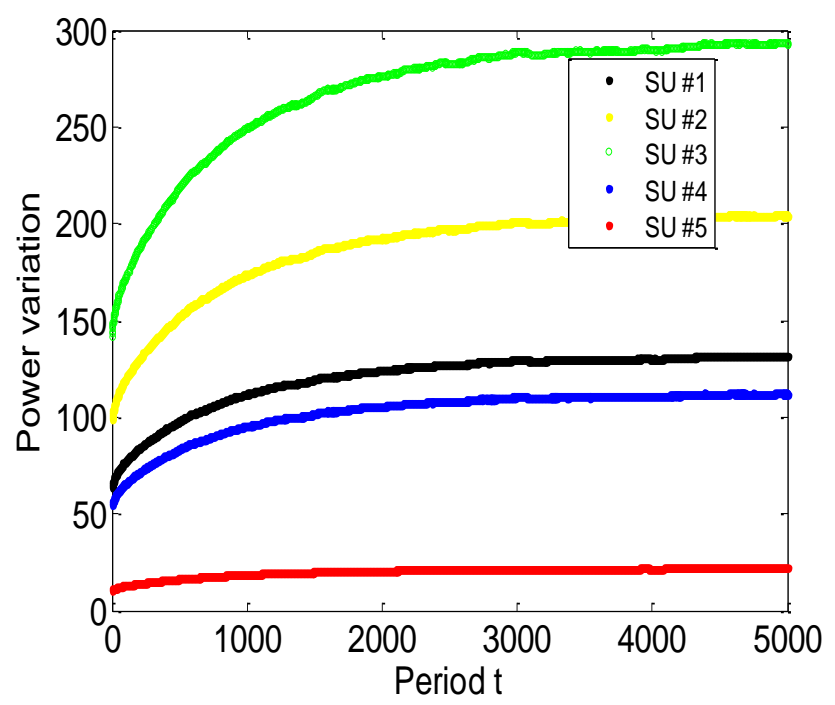

Fig. 3. SUs estimated power over period t.

The power transmitted by the SUs have been observed and evaluated. As can be seen from Fig. 3, it shows that the SUs are capable of maintaining their power level within the acceptable range. None of the SUs has exceeded the power threshold level and it proves how the developed scheme has been able to meet with the network constraint in order to achieve high performance and at the same keep in line with the constraints. The maximum achievable power transmitted by each SU after the network has completely converged is presented in Fig. 4.

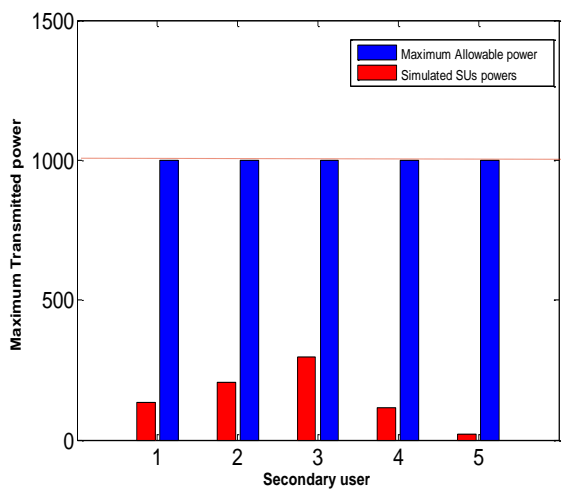

Fig. 4. Maximum allowable power limit for each user and SUs respective transmitted power. 


\section{B. Secondary Users Outage Probability Convergence}

In order to determine the respective outage probability of each SU, the outage probability was observed and represented in graphical form. As it can be seen from Fig. 5, the SU's have different outage probability as in real world scenario. For instance, SU3 has the highest outage probability and SU5 has the lowest outage probability when compared to the other SUs.

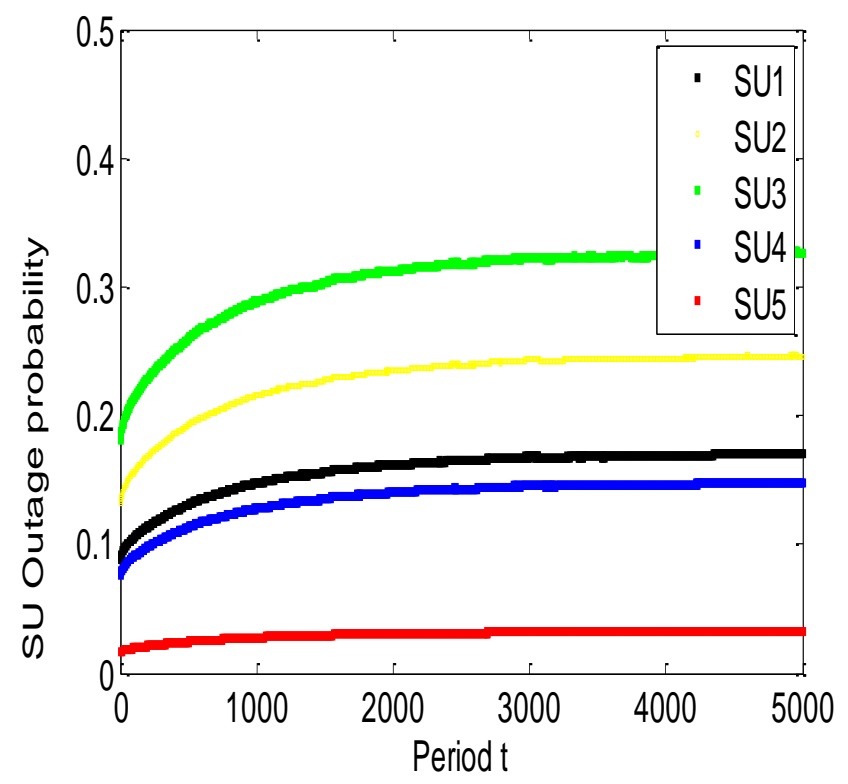

Fig. 5. Secondary users outage probability.

The main primary motive of considering the outage probability is to ensure that the QoS requirements for both the PU and SU have been achieved. This clearly shows the dramatic need to select best possible transmission power and outage probability. In a nutshell, it is very important to note that only the outage probability of SU5 is below the PU outage probability. This is due to the fact that there is need to provide QoS to SUs in order to ensure effective spectrum ulitization by the secondary user within the cognitive radio network. The value of the PU outage probability has stragically been chosen to ensure that the required QoS by both the primary and secondary users to accomplish the targeted network performance.

\section{Overall SUs Outage Probability Convergence}

In Fig. 6, the developed distributed QoS constraint based power control scheme has been tested based on the maximum number of SUs within the network which implies that the QoS of the PU and constraints have been reached. It clearly shows that the developed scheme can conveniently can be able to determine the outage probability precisely when all the SUs estimated their respective outage probability precisely. Once this has been accomplished, the scheme is said to have had converged to optimal. The overall SUs outage probability steady converges toward the threshold value which has been set to $10 \%$. This indicates that the proposed scheme can be able to adapt with time. It starts to converges from 0 and increase exponentially with time $\mathrm{t}$ as shown in Fig. 6.

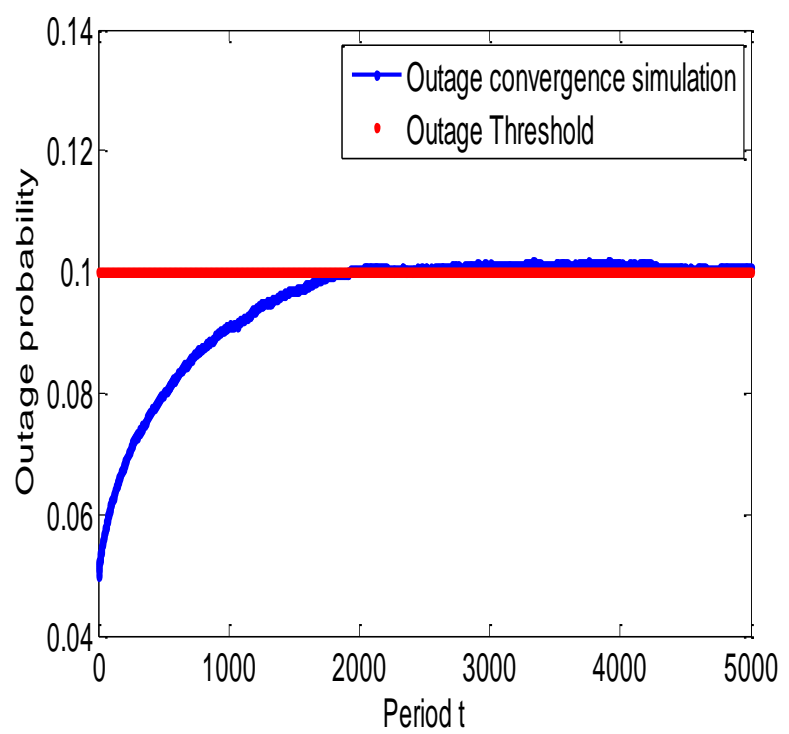

Fig. 6. Distributed QoS constraint based power control scheme outage probability convergence.

Interestingly, it is clear that the ability to utilized the spectrum effectively depend on the precision of the information received by SUs, but in our case no any information is receives from other SUs. Relying more on other SUs would not assist in situation where no any other SU is present within the coverage of the cognitive radio network which makes it difficult for the SU to utilize the network. Hence forth, the need for decentralize scheme is very crucial and extremely important under such conditions. As it can be noticed that the network performance increases rapidly at the initial stage and it become steady as the distributed QoS constraint based power adaptation algorithm converge. It is mainly due to the constraint put in place on the scheme. It has been observed that the proposed decentralize power control algorithm is more effective and reliable since the resources are distributed across the network.

\section{Optimal Network Utility Performance Comparison}

In this section, the SUs utility performance comparison test was conducted to verify the impact of adaptation in enhancing the network utilization in multi-user cognitive radio network. As can be seen from Fig. 7, the proposed distributed QoS constraint based power control scheme achived high network utilization when compared to the situation when no adaptation strategy has been used. Hence, it can be clearly seen that the proposed adaptation strategy improves the utility performance. Interestingly, the proposed distributed QoS constraint based power adaptation scheme converges faster toward theoptimal network utility when compared to without adaptation scheme. It is very obvious that the proposed adaptation scheme has consistently improves the utility over the period of time $t$.

The efficiency of the proposed scheme increases with increase in time and it converges faster. After the scheme converged, the network utilization remains relatively constant as it can be seen in Fig. 7. Howeover, the power at which every secondary user transmit is fully controlled in order to 
ensure that the users coexist together - without affecting the performance of other SUs. Moreover, there is need for effective power management as the network condition dynamically changes in order to ensure fairness to both the primary and secondary users. The proposed scheme achieve more better performance with the update, but the power consumption of wireless applications is growing exponentially which requires highly efficient techniques for power control. The fundamental basic findings of this research will be used in developing more better power control strategies which control the transmitted power, and it will improve the network capacity and reduces network cost. Therefore, the use of nature-inspired approaches for energy-efficient resource allocation in cognitive radio network will be employ and fully exploited.

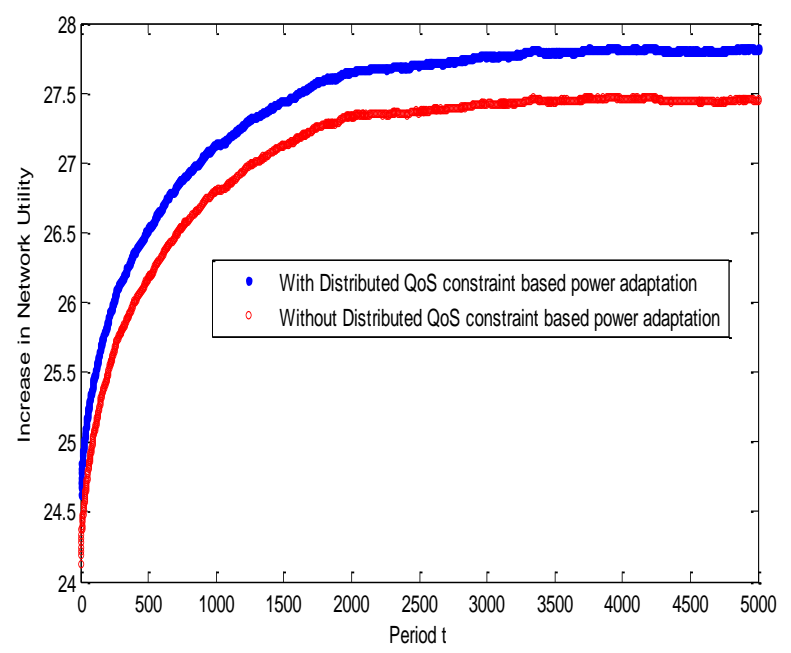

Fig. 7. Distributed QoS constraint based power control scheme with and without adaptation.

On a final note, both high convergence and network utility have been achieved using the proposed distributed QoS constraint based power control scheme. Undoubtedly, as the number SU increases, the network complexity will increase which eventually makes the decision more difficult and challenging. More intensive research work need to be conducted on how to enhance the performance of the proposed scheme further using energy-efficient nature-inspired optimization algorithms for effective power control and management in cognitive radio network.

\section{CONCLUSION}

In this paper, it primarily focuses on the development of distributed QoS constraint based power control scheme in order to maximize network utility in multi-user cognitive radio network. The optimization problem has been formulated and solved to ensure coexistence between the primary and secondary users within the network and subsequently it enhances the secondary user utility. This work has considered the power constraint from the PU so that the SU will sense and utilize the spectrum within the thresholds limits in order to comply with the QoS requirements. Numerically, it has been shown that the proposed scheme effectively improves the performance of the multi-user cognitive radio network. This eventually helps in effective and efficient sensing and management of the SUs with relative ease and sophistication. The distributed QoS constraint based power control scheme has been proposed and its performance is validated numerically. Our future will primarily focus on the usage nature-inspired approach to cooperatively enhance the performance of the network. The behavior of swarms will be mimic and adapted in order to effectively coordinate and manage the multi-users cognitive radio network to achieve higher performance and to reduce the network complexity.

\section{ACKNOWLEDGMENT}

The author would like to thank all those who contributed toward making this research successful. Also, I would like to thanks the reviewers for their insightful and valuable comment. This work is supported by the Deanship of Scientific Research (DSR), King Abdulaziz University, Saudi Arabia, under grant No. 830-129-D1437. The author is grateful to the DSR for their technical and financial support.

\section{REFERENCES}

[1] J. Mitola and G. Q. Maguire, "Cognitive radio: Making software radios more personal," IEEE Personal Communications, vol. 6, 1999.

[2] E. C. Y. Peh, Y.-C. Liang, Y. L. Guan, and Y. Zeng, "Optimization of cooperative sensing in cognitive radio networks: A sensing-throughput tradeoff view," IEEE Transactions on Vehicular Technology, vol. 58, 2009.

[3] Z. Quan, S. Cui, A. H. Sayed, and H. V. Poor, "Optimal multiband joint detection for spectrum sensing in cognitive radio networks," IEEE Transactions on Signal Processing, vol. 57, 2009.

[4] H. Zhang, C. Jiang, N. C. Beaulieu, X. Chu, X. Wen, and M. Tao, "Resource allocation in spectrum-sharing OFDMA femtocells with heterogeneous services," IEEE Transactions on Communications, vol. 62, 2014.

[5] J. C. F. Li, W. Zhang, and J. Yuan, "Opportunistic spectrum sharing in cognitive radio networks based on primary limited feedback," IEEE Trans. Commun., vol. 59, 2011.

[6] R. Zhang, "On active learning and supervised transmission of spectrum sharing based cognitive radios by exploiting hidden primary radio feedback," IEEE Trans. Commun., vol. 58, 2010.

[7] Y. He and S. Dey, "Power allocation in spectrum sharing cognitive radio networks with quantized channel information," IEEE Trans. Commun., vol. 59, 2011.

[8] G. Foschini and Z. Miljanic, "A simple distributed autonomous power control algorithm and its convergence," IEEE Transactions on Vehicular Technology, vol. 42, 1993.

[9] R. D. Yates, "A framework for uplink power control in cellular radio systems," IEEE Journal on Selected Areas in Communications, vol. 13, 1996.

[10] J. Huang, R. Berry, and M. Honig, "Distributed interference compensation for wireless networks," IEEE Journal on Selected Areas in Communications, vol. 24, 2006.

[11] M. Chiang, "Balancing transport and physical layers in wireless multihop networks: jointly optimal congestion control and power control," IEEE Journal on Selected Areas in Communications, vol. 23, 2005.

[12] S. Kandukuri and S. Boyd, "Optimal power control in interference limited fading wireless channels with outage-probability specifications," IEEE Transactions on Wireless Communications, vol. 1, 2002.

[13] M. Chiang, C. W. Tan, D. Palomar, D. O’Neill, and D. Julian, "Power control by geometric programming," IEEE Transactions on Wireless Communications, vol. 6, 2007.

[14] W. Ren, Q. Zhao, and A. Swami, "Power control in cognitive radio networks: how to cross a multi-lane highway," IEEE Journal on selected area in communications, vol. 27, 2009. 
[15] S. Srinivasa and S. Jafar, "Soft sensing and optimal power control for cognitive radio," IEEE Global Telecommunications Conference(GLOBECOM), 2007.

[16] W. Wang, Y. Cui, T. Peng, and W. Wang, "Noncooperative power control game with exponential pricing for cognitive radio network," in Proc. 2007 IEEE Vehicular Technology, 2007.

[17] S Ni.Q. Sun, J.X. Di, and W.M.. "Distributed power control based on convex optimization in cognitive radio networks," In International
Conference onWireless Communications and Signal Processing (WCSP), Suzhou, 2010.

[18] Y.N. Mei, Y.H. Lu, X.M. Mu, and X. Liu. "Distributed fast convergent power allocation algorithm in underlay cognitive radio networks," Wireless Internet, vol 98, 2012.

[19] C.G. Yang, J.D. Li, and Z. Tian. "Optimal power control for cognitive radio networks under coupled interference constraints: a cooperative game-theoretic perspective," IEEE Transactions on Vehicular Technology, vol 59, 2010. 